\title{
Thinking about the mystery of consciousness and panpsychism
}

\author{
Ho Manh Tung \\ Ritsumeikan Asia Pacific University \\ Beppu, Oita, Japan
}

August 26, 2020

Recently I read Conscious, a short book on the problem of consciousness by Annaka Harris ${ }^{1}$. The book offers a concise summary of the history of philosophical thoughts and the recent scientific advances in addressing the problem of consciousness. It has felt more relevant because I can observe my newborn child growing in his awareness of the world day by day.

When you talk about consciousness, there are a few names that must be dropped: Thomas Nagel with his famous essay, "What is it like to be a bat?" 2; David Chalmers with his definition of the "hard problem of consciousness"3; Sir Roger Penrose with his book "The Emperor's New Mind," where he provides the first account of consciousness based on quantum physics and theory of computation 4 . More recently, with advances neuroscience and computational psychology, we have emerging theories such as Donald Hoffman's "interface theory of perception" 5 , or attempts to explain consciousness by invoking homeostasis by Antonio Damasio 6,7, or the free energy principle and extended homeostasis' account of consciousness by Mark Solms ${ }^{8}$.

How do we begin to assess the claims in each of these theories? Here, I think there is a role for the philosophy of science; for example, Karl Popper's falsificationism ${ }^{9}$ or David Deustch's criteria of a good explanation ${ }^{10}$. Each of the theories must be judged based on whether they are internally coherent, meaning each explanatory part is interlocking and hard to vary; and whether they yield testable predictions.

There are many theories of consciousness based on natural selection and biology. It seems to me although consciousness seems related to natural selection, as all living creatures seem to be conscious, in the case of the smart computers, natural selection might not play any role at all. It is not clear how the theories of consciousness grounded on biological phenomenon would help us determine whether computers are conscious. 
Then there is so much hope for a theory of consciousness to emerge from a complete correlation mapping between the first-person subjective experiences and the third-person accounts of neuronal activities. Yet, even here, for me, the map would not explain away the fundamental mystery of how non-conscious matters give rise to consciousness, the hard problem of consciousness.

Another way of explaining consciousness is how it is related to "problem-solving" and "creativity." It seems to me to solve a problem; one must first be aware of the problem; even a mosquito must be aware of a danger to get out of the way. But how much consciousness depends on the problem of survival and reproductive success? It seems that most problems can be solved algorithmically, thus requiring no consciousness at all. I can conceive of a humanoid robot, solving all the problems relevant to a human: seeking foods, shelters, status, etc., yet there is "nothing it is like to be that humanoid robot," there is no light of consciousness inside.

Thus, we must ask whether consciousness is a biological or computational or a physical problem. Undoubtedly, a physical theory of consciousness will be more encompassing. And it seems the biological and computational accounts of consciousness will account for species-specific or cultural-specific ${ }^{11,12}$ or context-specific types ${ }^{13}$ of conscious experience.

This leads me to the surprising thing I learn from reading Annaka Harris's Conscious: panpsychism seems to be one of the most coherent theories of consciousness; even it sounds crazy at first. Panpsychism posits consciousness is a fundamental property of the physical world. All matters possess some degree of consciousness. Complex consciousness of, for example, humans are the results of complex combination and interaction of simple consciousness of matters. Critics of this theory have raised a challenge of combination, the so-called "combination problem of panpsychism," 14 meaning how panpsychism accounts for the process of combining micro-consciousness to become a macro-consciousness. But the problem with this way of thinking is the concept of subject is advertently smuggled in. All there is consciousness and its contents (feeling of heats, pressure, temperature, pain, pleasure, etc.), it is an ever-changing and fleeting field of experiences. There is no subject. Thus, there should be no problem when two collection of conscious contents merge, all there will be is the process of contents coming and going out of the light of consciousness. 


\section{References}

1. Harris, A., 2019. Conscious: A brief guide to the fundamental mystery of the mind. HarperCollins, New York.

2. Nagel, T., 1974. What is it like to be a bat?. The philosophical review, 83(4), 435-450.

3. Chalmers, D.J., 1995. Facing up to the problem of consciousness. Journal of Consciousness Studies, 2(3), 200-219.

4. Roger, P., 1990. The Emperor's New Mind. Oxford University Press, Oxford.

5. Hoffman, D.D., Singh, M., and Prakash, C., 2015. The interface theory of perception. Psychonomic Bulletin \& Review, 22(6), 1480-1506.

6. Damasio, A., 2019. The strange order of things: Life, feeling, and the making of cultures. Vintage, London.

7. Man, K., and Damasio, A., 2019. Homeostasis and soft robotics in the design of feeling machines. Nature Machine Intelligence, 1(10), 446-452.

8. Solms, M., 2019. The hard problem of consciousness and the free energy principle. Frontiers in Psychology, 9, 2714.

9. Popper, K., 2014. Conjectures and refutations: The growth of scientific knowledge. Routledge, UK.

10. Deutsch, D., 2011. The beginning of infinity: Explanations that transform the world. Penguin, UK.

11. Vuong, Q. H., Ho, M. T., Nguyen, H. K. T., Vuong, T. T., Tran, T., Hoang, K. L., ... \& La, V. P. (2020). On how religions could accidentally incite lies and violence: Folktales as a cultural transmitter. Palgrave Communications, 6(1), 82.

12. Vuong, Q. H., Bui, Q. K., La, V. P., Vuong, T. T., Nguyen, V. H. T., Ho, M. T., ... \& Ho, M. T. (2018). Cultural additivity: behavioural insights from the interaction of Confucianism, Buddhism and Taoism in folktales. Palgrave Communications, 4(1), 143.

13. Huu, N.V., Hoang, V.Q., Ngoc, T.M. (2005). Central limit theorem for functional of jump Markov processes. Vietnam Journal of Mathematics, 33(4), 443-461 
14. Coleman, S. (2014). The real combination problem: Panpsychism, micro-subjects, and emergence. Erkenntnis, 79(1), 19-44. 